\title{
A note on closedness of algebraic sum of sets
}

\author{
Hubert Przybycień
}

Faculty of Mathematics and Computer Science, Adam Mickiewicz University Umultowska 87, 61-614 Poznań, Poland

E-mail: hubert@amu.edu.pl

\begin{abstract}
In this note we generalize the fact that in topological vector spaces the algebraic sum of closed set $A$ and compact set $B$ is closed. We also prove some conditions that are equivalent to reflexivity of Banach spaces.
\end{abstract}

2010 Mathematics Subject Classification. 46A22. 46N10

Keywords. Closed convex sets.

\section{Inroduction}

Let $X$ be a Hausdorff topological vector space. By $\mathcal{C}(X)$ we denote the family of all closed subsets of $X$ and by $\mathcal{K}(X)$ the family of all compact subset of $X$. For a nonempty subsets $A, B \subset X$ we define the algebraic sum (Minkowski sum) as follow

$$
A+B=\{a+b: a \in A, b \in B\} .
$$

It is wll known that if $A, B$ are closed sets then $A+B$ need not to be closed, but also it is known that if $A \in \mathcal{C}(X)$ and $B \in \mathcal{K}(X)$ then $A+B \in \mathcal{C}(X)$. In this note we prove the last result with some more abstract point of view.

\section{Reflexive spaces}

In this section we give some results which show the connections of closedness of algebraic sum of sets with reflexivity of Banach spaces.

Theorem 2.1. Let $X$ be a Banach space and let $B$ be the closed unit ball in $X$. Then $X$ is a reflexive Banach space if and only if for every closed convex and bounded subset $A$ of $X$ the algebraic sum $A+B$ is closed.

Proof. (Necessity.) Assume that $X$ is a reflexive Banach space, then every closed bounded and convex set is a weakly compact. Since algebraic sum of two weakly compact sets is again weakly compact we conclude that $A+B$ is a weakly compcact set, and therefore closed.

(Sufficiency.) Assume that $X$ is not reflexive Banach space. Then by Theorem of James there exists a continuous linear functional

$f: X \rightarrow \mathbb{R}$, such that $\|f\|=1$ and $f(x)<1$ for every $x \in B$.

Let

$$
A=\{x \in X: f(x) \geqslant 1,\|x\| \leqslant 2\}
$$

Then $A$ is a closed bounded and convex set but $A+B$ is not closed.

Q.E.D.

Tbilisi Mathematical Journal 9(2) (2016), pp. 71-74.

Tbilisi Centre for Mathematical Sciences.

Received by the editors: 14 February 2016.

Accepted for publication: 16 September 2016 
Now using the theorem 1 we prove the following theorem

Theorem 2.2. Let $X$ be a Banach space and denote by $\tau_{s}$ the norm topology. Then $X$ is a reflexive Banach space if and only if there exists a linear Hausdorff topology $\tau$ on $X$ such that $\tau \subset \tau_{s}$ and every closed convex and bounded subset of $X$ is compact in the topology $\tau$.

Proof. (Necessity.) If $X$ is a reflexive Banach space then we can take $\tau$ to be equal the weak topology on $X$.

(Sufficiency.) Suppose that every closed convex and bounded subset of $X$ is compact in the topology $\tau$. Then for any closed convex and bounded set $A$ the algebraic sum $A+B$, (where $B$ is a closed unit ball in $X$ ) is compact in $\tau$, and hence closed in $\tau$, but since $\tau \subset \tau_{s}$ it is also closed in $\tau_{s}$. Thus by theorem 1 the space $X$ is reflexive.

Q.E.D.

Definition 2.3. Let $X$ be a topological vector space and $A, B \subset X$. We say that the sets $A$ and $B$ can be strictly separated by hyperplane if there exists a continuous functional $f: X \rightarrow \mathbb{R}$, and real numbers $u, v$ such that

$$
f(x)<u<v<f(y) \text { for all } x \in A, y \in B .
$$

It is well known that in locally convex topological vector spaces a closed convex sets can be strictly separated from disjoint compact convex sets by a hyperplane.

The next theorem show the connections between a reflexivity and separation.

Theorem 2.4. Let $X$ be a Banach space. Then $X$ is reflexive Banach space if and only if every two disjoint closed bounded and convex sets can be strictly separated by a hyperplane.

Proof. (Necessity.) Let $X$ be a reflexive Banach space and let $A, B$ be a two nonempty disjoint closed convex and bounded sets. Then $X$ with the weak topology $\tau_{w}$ is a locally convex space in which the sets $A, B$ are compact and thus by a separation theorem $A, B$ can be strictly separated by a hyperplane in $\left(X, \tau_{w}\right)$. Surely the sets can be also separated by a hyperlane in $X$ since every weak continuous linear functional is also continuous in the norm topology.

(Sufficiency.) Assume that every two disjoint closed bounded and convex sets can be strictly separated by a hyperplane. Let $A, B$ be two disjoint closed bounded and convex sets, we prove that in this case the set $A-B$ is closed. To prove this assume contrary that $A-B$ is not closed. Without loss of generality we may assume that $0 \in \overline{A-B} \backslash(A-B)$. By assumption there exists a continuous linear functional $f: X \rightarrow \mathbb{R}$ such that

$$
f(x)<u<v<f(y) \text { for all } x \in A, y \in B,
$$

for some $u, v \in \mathbb{R}$.

Therefore

$$
f(w)<u-v \text { for all } w \in A-B,
$$

hence

$$
f(w) \leqslant u-v<0 \text { for all } w \in \overline{A-B},
$$


thus $0 \notin \overline{A-B}$ and we get a contradiction.

So we have just proved that for any two disjoint closed bounded and convex sets $A, B$, the set $A-B$ is closed.

Now let $A, B$ be any two closed convex and bounded sets. Then there exists a vector $x \in X$ such that the sets $x+A$ and $B$ are disjoint, and hence (by what we have just proved above) the set $(x+A)-B$ is closed. But then the set $[(x+A)-B]-x=A-B$ is closed too.

Finally from the equality $A+B=A-(-B)$ we obtain that the sum $A+B$ is closed for any two closed convex and bounded set therefore by theorem 1 the space $X$ is a reflexive Banach space.

Q.E.D.

The last theorem in this section gives also a condition which is equivalent to reflexivity for Banach spaces.

Theorem 2.5. A Banach space $X$ is reflexive if and only if every closed and convex set has an element with minimal norm.

Proof. (Necessity.) Assume that $X$ is a reflexive Banach space and let $A$ be a nonempty closed and convex set. Without loss of generality we may assume that $0 \notin A$. Let $a=\inf _{x \in A}\|x\|$ we'll show that there is an element $u \in A$ such that $\|u\|=a$. To prove this let

$$
B_{n}=\left\{x \in X:\|x\| \leqslant a+n^{-1}\right\}
$$

then the sets $C_{n}=B_{n} \cap A$ form a descending sequence of nonempty closed convex and bounded and hence weakly compact and convex sets. Thus

$$
C=\bigcap_{n} C_{n} \neq \varnothing
$$

and it is easy to observe that for $v \in C$ we have $\|v\|=a$.

(Sufficiency.) Assume that every closed and convex set has an element with minimal norm. Let $f: X \rightarrow \mathbb{R}$ be a continuous linear functional with $\|f\|=1$. Then the set $A=\{x \in X$ : $f(x) \geqslant 1\}$ is closed and convex therefore by assumption it has an element $x_{0}$ with minimal norm. It is easy to observe that $\left\|x_{0}\right\|=1$ and thus $f$ attains its maximum on unit ball. Hence by theorem of James $X$ is reflexive Banach space.

Q.E.D.

\section{Closedness of algebraic sum}

In this section we prove a theorem which is a generalization of the fact that in Hausdorff topological vector space the algebraic sum of closed set $A$ and compact set $B$ is closed.

Theorem 3.1. Let $X, Y, Z$ be Hausdorff topological spaces and let $f: X \times Y \rightarrow Z$ be a function such that:

(a) $f$ is continuous, and for every $y \in Y$ the function $f(\cdot, y)$ is an injection.

(b) there exists a continuous function $\varphi: Y \times Z \rightarrow X$ such that $f(\varphi(y, z), y)=z$ for all $(y, z) \in Y \times Z$. 
Assume that $A \subset X \times Y$ is a closed set such that:

(c) the projection $\pi_{Y}(A)=\{y \in Y:(x, y) \in A\}$ of the set $A$ is compact.

Then the image $f(A)=\{z \in Z: z=f(x, y)$ for some $(x, y) \in A\}$ of the set $A$ is closed.

Proof. Let $z_{\alpha} \in f(A), \alpha \in \Lambda$ be an MS-sequence tending to $z_{0}$. Then there exists a MSsequences $x_{\alpha}, y_{\alpha}, \alpha \in \Lambda$ such that $\left(x_{\alpha}, y_{\alpha}\right) \in A$ and $z_{\alpha}=f\left(x_{\alpha}, y_{\alpha}\right)$. Therefore $x_{\alpha} \in$ $\pi_{X}(A), y_{\alpha} \in \pi_{Y}(A)$, but since $\pi_{Y}(A)$ is compact there exists a MS-subseqence $y_{\beta}, \beta \in \Sigma$ of the MS-sequence $y_{\alpha}, \alpha \in \Lambda$ such that $y_{\beta} \rightarrow y_{0} \in \pi_{Y}(A)$. Moreover by the continuity of $\varphi$ we get $x_{\beta}=\varphi\left(y_{\beta}, z_{\beta}\right) \rightarrow \varphi\left(y_{0}, z_{0}\right)=x_{0} \in X$. But $A$ is closed and $\left(x_{\beta}, y_{\beta}\right) \in A,\left(x_{\beta}, y_{\beta}\right) \rightarrow\left(x_{0}, y_{0}\right)$ thus $\left(x_{0}, y_{0}\right) \in A$. Therefore $f\left(x_{\beta}, y_{\beta}\right) \rightarrow f\left(x_{0}, y_{0}\right) \in f(A)$. But $f\left(x_{\beta}, y_{\beta}\right) \rightarrow z_{0}$ and thus $z_{0}=f\left(x_{0}, y_{0}\right) \in f(A)$ and the proof is complete.

Q.E.D.

Corollary 3.2. Let $(G,+)$ be a Hausdorff topological group and let $B, C \subset G$, be subsets such that $B$ is closed and $C$ is compact. Then the algebraic sum $B+C$ is closed.

Proof. To prove this it is enough to take in theorem $5: X=Y=Z=G, A=B \times C$, $f(x, y)=x+y, \varphi(y, z)=z-y$.

Q.E.D.

\section{References}

[1] A. Alexiewicz, Functional Analysis (1969) (in Polish)

[2] J. Dieudonne, Sur la sparation des ensembles convexes, Math. Ann. 163 (1966)

[3] Robert C. James, Reflexivity and the sup of linear functionals, Israel J. Math 13 (1972), $3-4$

[4] W. Rudin, Functional Analysis, McGraw-Hill Book Company, New York, 1973 